\title{
Study of hereditary thrombophilia (factor $V$ leiden and protrombine 20210) in a population of women in fertile age who suffered a thromboembolic event studied in the tucumán public health laboratory
}

\begin{abstract}
The thromboembolic event (TE) is a public health problem associated with significant morbidity and mortality, whose management includes the evaluation of the hypercoagulable state of the patient, especially in young individuals. The most frequent hereditar y thrombophilias are the Factor V Leiden polymorphism (FVL) and the Prothrombin 20210 (PT20210). In the general Caucasian European population, the prevalence of VLF is 3 to $7 \%$ and $1-4 \%$ for PT20210 and in patients with a first TE it is 20-50\% for FVL and 5- 10\% for PT20210. Studies performed in our country revealed $2.9 \%$ of heterozygous carriers for FVL and 2.6\% for PT20210 in the general population, similar to that described for southern Europe, while other authors reported $10 \%$ and $6.3 \%$ for FVL and PT20210 re spectively in individuals with a first TE. The objective of this study was to investigate FVL and PT20210 in young women, of childbearing age, who suffered a TE without apparent risk factors and were referred to the Public Health Laboratory of Tucumán in 2015 and 2016. A descriptive observational study was conducted cross section in 39 women. It was detected that $2.5 \%$ (1/39) were heterozygous for FVL while 5.7\% (2/39) for PT20210.

Our study includes patients living in the province of Tucumán, where we obs erved that the prevalence of mutations was lower than those described in other regions of the country, possibly reflecting the different ethnic composition; nevertheless, these genetic alterations are present in our province and Scientific societies recomm end the study of these in selected patients. These women could face circumstantial prothrombotic risk factors such as pregnancy, trauma, prolonged immobilization, hormonal treatments that would predispose to a new TE, for which the investigation of these $\mathrm{g}$ enetic variants is necessary for their prevention.
\end{abstract}

Keywords: thrombophilia,venous thromboembolism, molecular biology, risk factor, resistance to protein $\mathrm{C}$ activated
Volume 13 Issue 2 - 2020

\author{
Rios Mariana, Fernández Zenoff, Verónic, \\ Hayward Cecilia, Suarez A Viviana, Rossi \\ Eleonora B \\ Department of Biochemical, Laboratory of Public Health, \\ Argentina
}

Correspondence: Rios Mariana, Department of Biochemical, Laboratory of Public Health, Argentina,

Email marianariosar@yahoo.com.ar

Received: March 19,2020 | Published: April 16, 2020
Abbreviations: ET, thromboembolic event; FVL, factor V leiden; PT 20210, prothrombin 20210; DVT, deep vein thrombosis; CVA, stroke; NMR, Nuclear Magnetic Resonance; WHO, world health organization; DB-LSP, biochemical department public health laboratory; PCR, polymerase chain reaction

\section{Introduction}

Thrombophilia is a disorder of the hemostatic mechanism where an abnormal predisposition to thrombosis is demonstrated. It can manifest as a spontaneous thromboembolic event or of a severity disproportionate to the stimulus, in the venous and occasionally arterial territory, with a variable tendency to recur, in unusual locations; it rarely occurs in young patients and may be accompanied by a family thrombotic history. The presence of this predisposing factor does not necessarily imply the development of a thrombotic event. Thrombotic disease is multifactorial as a result of the conjunction of various factors, genetic, environmental, and acquired conditions that determine individual clinical expression. ${ }^{1 \backslash}$

Venous thromboembolic disease (VTE) is a vascular disease with a high impact on morbidity and mortality worldwide. One in 4 deaths worldwide is related to thrombosis. ${ }^{2}$ In Argentina it is estimated that more than 60 thousand people suffer a Venous Thromboembolism (VTE) per year, of which 48 thousand manifest it as Deep Venous Thrombosis (DVT), and about 15 thousand as Pulmonary Thromboembolism (PET or Pulmonary Embolism), taking into account that the mortality rate of the latter is up to $30 \%$, this translates into some 4,300 deaths per year. ${ }^{3}$ The risk of thrombosis in women increases during pregnancy and the puerperium, during taking oral contraceptives, with hormonal treatment in ovarian stimulation for assisted fertilization and in hormone replacement therapy during menopause.

International consensus suggests studying thrombophilia in young patients who had venous, arterial, or PE thrombosis. The WHO defines hereditary thrombophilia as a genetically determined tendency to venous thromboembolism. ${ }^{4}$ Both dominant abnormalities and milder combinations of defects in others may be clinically apparent with early age of onset, frequent recurrences, or a family history of thrombosis. The family history of first degree thrombosis or gestational complications associated with thrombophilia is also situations to be considered. The recurrence of a thrombotic event at 2 years is $17 \%$ and increases to $30 \%$ at 8years. ${ }^{5}$ Defects in the coagulation Factor V molecule have been shown to interfere with the inhibitory regulatory mechanism of activated Protein C, contributing to the pathogenesis of thrombosis. These defects in the Factor V molecule are associated with 
a point mutation in exon 10 of the gene where a guanine is replaced by an adenine at position 1691, giving rise to Factor V Leiden. ${ }^{6,7}$

Prothrombin or Coagulation Factor II is the precursor to thrombin, a key enzyme in the coagulation mechanism. A genetic variant (prothrombin 20210) in the 3 'untranslated region of the gene (substitution of guanine for adenine at position 20210) leads to increased synthesis of prothrombin as a consequence of increased efficiency of the messenger RNA, which is associated Factor V Leiden is the most frequent genetic condition associated with VTE, the prevalence of heterozygotes in the FVL is $5-8 \%$ in the Caucasian population, with a frequency of $18 \%$ in clinical series of VTE and 30 to $60 \%$ in patients with a family history of thrombophilia. ${ }^{8}$ Heterozygous individuals are 7 times more at risk of having a VTE compared to the general population; while in homozygotes the risk is 80 times greater.

The prevalence of heterozygous PT20210 carriers in the general population is 1 to $4 \%$, in patients who suffered a thrombotic event for the first time, it was found in $6.2 \%$ and in $18 \%$ of individuals with a family history of thrombosis. This mutation is associated with a 2.8 fold increase in the risk of thrombosis in the general population. For this reason, prevention is key, especially in those people who are at increased risk of ETV. ${ }^{9}$

\section{General objective}

The objective of the present work was to investigate the hereditary thrombophilic factors (FVL and/or PT 20210) of female patients of childbearing ages who suffered a thromboembolic event and who were referred to the Hemostasis Section of the Laboratory or Public Health (LSP) of San Miguel de Tucumán.

\section{Specific objective}

a. Tune up Molecular Biology techniques for the detection of FVL and PT20210 mutations.

b. Investigate if the aforementioned patients are carriers of Factor $\mathrm{V}$ Leiden and Prothrombin 20210 mutations.

\section{Materials and methods}

\section{Type of study and design}

Descriptive observational cross-sectional study.

\section{Population and Sample}

The research project was carried out in the facilities of the Public Health Laboratory of the province of Tucumán, in the Hemostasis section. The records of 6906 patients who attended the laboratory from January 2015 to August 2017 were analyzed.

Inclusion criteria: young women of childbearing age who suffered a duly registered thrombotic event, outside of a pregnancy.

Exclusion criteria: patients with some underlying disease.

87 women of childbearing age who had suffered a thrombotic event (DVT, PE, or ischemic stroke) were preselected; of which 41 were presented in the LSP, each patient filled out a clinical history and they also signed an informed consent where they authorized us to study them once the objectives and scope of the research project were explained to them, which had been approved by the Ethics Committee of the Ministry of Health of the province of Tucumán. 2 patients were separated because at the time of evaluating their medical studies (Doppler ultrasound or MRI) and the treatment they had carried out, it was concluded that they had not suffered an ET. The blood samples of the 39 resulting patients were taken with an 8-hour fast and received in tubes with EDTA as anticoagulant and frozen at $-20^{\circ} \mathrm{C}$ until processing. Table 1 details the characteristics of the studied population.

For the detection of the mentioned polymorphisms, genomic DNA was used, which was obtained by using a commercial Nucleo Spin Blood extraction kit (Genomic DNA from blood). Subsequently, work was carried out on the development of the Polymerase Chain Reaction (PCR) for the two polymorphisms, whose conditions were optimized for our laboratory. Amplification of Factor V and Prothrombin genes was performed using the following primer or primers: ${ }^{12}$

\section{For Factor V: (Forward) 5' TGC CCA GTG CTT AAC AAG ACC A 3 \\ (Reverse) 5' CTT GAA GGA AAT GCC CCA TTA 3' \\ Prothrombin: (Forward) 5' TCT AGA AAC AGT TGC CTG GC} 3

\section{(Reverse) 5' ATA GCA CTG GGA GCA TTG AA * GC 3}

The first of the Prothrombin in the $3^{\prime}$ chain carries a nucleotide substitution (*), which, when combined with the PT G20210Amutation, generates a cleavage site with a restriction enzyme. ${ }^{9}$ Amplification of both genes was performed in a Veriti thermocycler from Applied Biosystems with a reaction volume of $50 \mu \mathrm{l}$ and the Taq polymerase reaction protocol was respected: $5 \mu 1$ of Buffer Green Dream Taq 10X, $1 \mu \mathrm{dN}$ TP $0.2 \mathrm{mM}, 1 \mu \mathrm{l}$ of each first $20 \mu \mathrm{M}$ (forward and reverse), $0.25 \mu 1$ of DreamTaq DNA Polymerase (Thermo scientific), $39.75 \mu 1$ of sterile water for molecular biology and $4 \mu$ l of DNA (the concentration of DNA had to be modified to obtain better performance in P CR).

The cycling conditions were

a. For the Factor $\mathrm{V}$ gene: initial denaturation, at $94{ }^{\circ} \mathrm{C} 3 \mathrm{~min} ; 35$ cycles of $1 \mathrm{~min}$ at $94{ }^{\circ} \mathrm{C}, 1 \mathrm{~min}$ at $56{ }^{\circ} \mathrm{C}$ and $1 \mathrm{~min}$ at $72{ }^{\circ} \mathrm{C}$ and final extension of $2 \mathrm{~min}$ at $72{ }^{\circ} \mathrm{C}$.

b. For the Prothrombin gene: initial denaturation, at $94^{\circ} \mathrm{C} 3 \mathrm{~min} ; 35$ cycles of $40 \mathrm{sec}$ at $94^{\circ} \mathrm{C}, 50 \mathrm{sec}$ at $58^{\circ} \mathrm{C}$ and $1 \mathrm{~min}$ at $72{ }^{\circ} \mathrm{C}$ and final extension of $2 \mathrm{~min}$ at $72{ }^{\circ} \mathrm{C}$.

As a negative control of amplification, sterile water was used and as a positive control, samples sent by the Haemostasis Laboratory of the Faculty of Exact and Natural Sciences of the UBA.

The amplification products were visualized on $1 \%$ agarose gels stained with SYBR ${ }^{\circ}$ Green I Nucleic Acid Gel Stain (Invitrogen), according to the manufacturer's specifications. The amplification products were 220 and 345 bp for Factor V and Prothrombin respectively. Subsequently, for the identification of mutations, the Restriction Fragment Length Polymorphism (RFLP) technique was performed . To do this, $20 \mu \mathrm{l}$ of amplified product were used, which were digested for 18 hours at $37^{\circ} \mathrm{C}$ with the following restriction enzymes:

i. Mnl I (Thermo scientific) for the Factor V amplification, where it was verified that the optimal concentration was $5 \mathrm{U}$ for every $10 \mu \mathrm{l}$ of amplification product.

ii. Hind III (Thermo scientific) for the Prothrombin amplification, where the optimal concentration was determined to be $20 \mathrm{U}$ for 
every $10 \mu \mathrm{l}$ of amplification product. The products of enzymatic digestion were run and visualized on a 2.5 and $3 \%$ agarose gel, respectively, and stained with SYBR ${ }^{\circledR}$ Green I Nucleic Acid Gel Stain (Invitrogen)

iii. The Mnl I enzyme recognizes 2 cleavage sites in the normal Factor $\mathrm{V}$ gene, generating 3 fragments of 116,67 and 37 base pairs. The Factor V Leiden (G1691A) mutation deletes a cut-off site in the altered gene, resulting in 2 fragments, 153 and $67 \mathrm{bp}$. Therefore, if the patient is heterozygous, the band pattern obtained will be 4 fragments: 153, 116, 67 and 37bp and if the patient is homozygous, only 2 fragments will be displayed: 153 and 67bp (Figure 1).

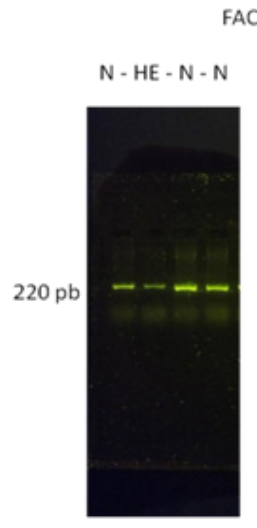

PCR

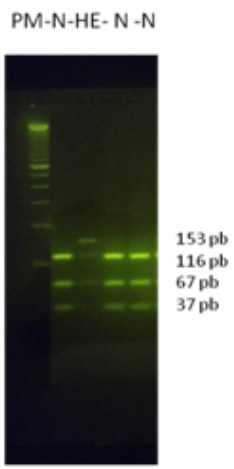

RFLP
Figure I The photograph on the left shows the PCR products, from different patients, obtained by amplifying a segment of the factor $V$ gene. On the right, the RFLP result is observed when using the restriction enzyme $\mathrm{Mnl} I$ in which the first lane corresponds to the 100bp (MW) molecular weight marker. Lanes 2, 4 and 5 show normal patients $(\mathrm{N})$ while lane 3 shows a heterozygous patient (HE).

For its part, the Hind III enzyme does not recognize cut sites in the normal prothrombin gene, leaving the amplified intact. But if one of the genes had the PT G20210A mutation, this in combination with the mutation incorporated in the PCR process, would generate a cutoff site which would lead to the appearance of 2 fragments: 322 and $23 \mathrm{bp}$. Therefore, if the patient were homozygous, 2 fragments would be visualized: 322 and $23 \mathrm{bp}$; while if the patient were heterozygous the band pattern would be 3 fragments: 345, 322 and 23 bp (Figure 2).

For statistical analysis of the data, the SPSS 9.0 program was used. For the study of quantitative variables, the arithmetic mean, median and range were calculated.

\section{Results}

In Table 1 the clinical characteristics of the detailed group of patients, women of childbearing age who had an ET who attended for evaluation to the DB-LSP of Tucuman. These patients come from different locations in the province and were treated during their ET in the system. At the time of sampling, the median age of the patients was 33years (17-42), the age at presentation of the first ET was 30 years (16-40). The predominant ET was Deep Vein Thrombosis 20/39, followed by Ischemic Cerebrovascular Accident 9/39 and thirdly Pulmonary Embolism Thrombus 6/39; the majority did not present sequelae but 6/39 had recurrence. Among the 39 patients with ET studied we found 1/39(2.6\%) heterozygous carrier for FVL and 2/39 (5.1\%) for PT20210 (Table 2). No homozygous or double heterozygous patient was detected for the mutations studied.
Protrombina 20210

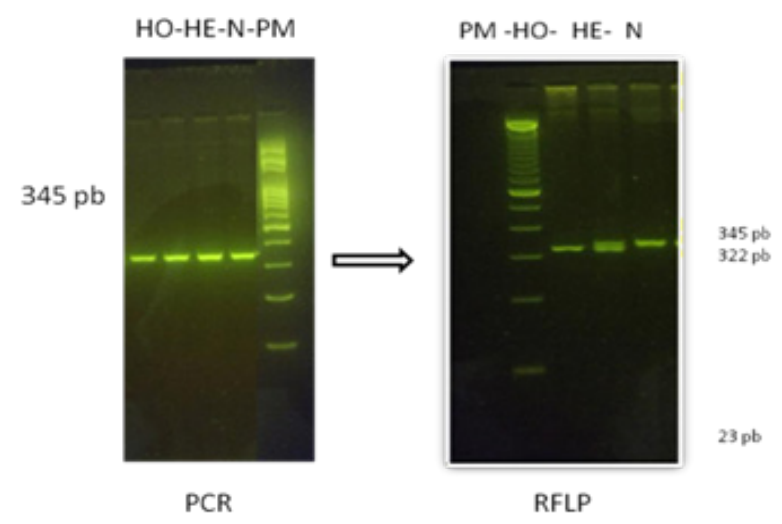

Figure 2 The photograph on the left shows the PCR products, from different patients that were obtained by amplifying a segment of the Prothrombin gene. On the right, the RFLP result is observed when using the restriction enzyme Hind III. In the first lane we have a 100 base pair molecular weight marker, in the second lane a control sample from a homozygous patient and in the third and fourth lanes we observe samples from a heterozygous and normal patient, respectively.

Table I Characteristics of the Population studied

\begin{tabular}{|c|c|}
\hline $\mathrm{n}$ & 39 \\
\hline \multicolumn{2}{|l|}{ Age(years) } \\
\hline Median & 33 \\
\hline Rank & $17-42$ \\
\hline \multicolumn{2}{|l|}{ Age of the first ET(years) } \\
\hline Median & 30 \\
\hline Rank & $16-40$ \\
\hline No. deeds(n) & 74 \\
\hline Medium & 2 \\
\hline rank & $0-6$ \\
\hline Children born alive(n) & 57 \\
\hline \multicolumn{2}{|l|}{ Spanish parental } \\
\hline Ancestry(n) & $10(25.6 \%)$ \\
\hline Italian & $5(12.8 \%)$ \\
\hline Creole & $12(3 \mid \%)$ \\
\hline Others & $4(10.3 \%)$ \\
\hline Don't know & $8(20.5 \%)$ \\
\hline \multicolumn{2}{|l|}{ Maternal ancestry(n) } \\
\hline Spanish & $12(3 \mid \%)$ \\
\hline Italian Others Don't know & $\mathrm{I}(2.6 \%)$ \\
\hline Creole & $16(4 \mid \%)$ \\
\hline Others & $2(5 \%)$ \\
\hline
\end{tabular}




\begin{tabular}{ll} 
Table Continued & 39 \\
\hline $\mathrm{n}$ & $8(20.5 \%)$ \\
\hline Don't know & 20 \\
TVP(n) & 2 \\
DVT + PET $(n)$ & 4 \\
Isolated PET(n) & 9 \\
LCA(n) & 4 \\
TV unusual sites $(n)$ & 6 \\
Recurrence $(n)$ & 5 \\
Antec. thrombosis $(n)$ &
\end{tabular}

Table 2 Genotypic distribution of the genetic variants studied in a population of women from Tucumán who suffered an ET in fertile age.

\begin{tabular}{lll}
\hline $\mathbf{n = 3 9}$ & FVL & P20210 \\
\hline Homozygous & $0 / 39$ & $0 / 39$ \\
Heterozygotes & $1 / 39(2.6 \%)$ & $2 / 39(5.1 \%)$ \\
Total & $3 / 39(7.7 \%)$ & \\
\hline
\end{tabular}

Table 3 Distribution of genetic variants studied locally and globally, differentiating between populations with and without thromboembolic event.

\begin{tabular}{|c|c|c|c|c|c|c|c|}
\hline & \multicolumn{3}{|c|}{ Population without ET } & \multicolumn{4}{|c|}{ Population with ET } \\
\hline & \multirow[b]{2}{*}{ *World } & \multirow[b]{2}{*}{ †Argentina } & \multirow[b]{2}{*}{ †Tucumán } & \multirow[b]{2}{*}{ *World } & \multirow[b]{2}{*}{$\ddagger$ Argentina } & \multicolumn{2}{|l|}{ Tucuman } \\
\hline & & & & & & $\begin{array}{l}\text { §ET during } \\
\text { feat }\end{array}$ & $\begin{array}{l}\text { ET without } \\
\text { deed }\end{array}$ \\
\hline $\begin{array}{l}\text { Heterozygous } \\
\text { FLV }\end{array}$ & $2-10 \%$ & $2.9 \%(|2 / 4| 8)$ & $0 \%(0 / 20)$ & $20-50 \%$ & $10 \%(19 / 192)$ & $0 \%(0 / 39)$ & $2.6 \%(1 / 39)$ \\
\hline $\begin{array}{l}\text { Heterozygous } \\
\text { P20210 }\end{array}$ & $1-4 \%$ & $2.6 \%(|I / 4| 8)$ & $5 \%(I / 20)$ & $5-10 \%$ & $6.3 \%(12 / 190)$ & $\begin{array}{l}0 \% \\
(0 / 39)\end{array}$ & $5.1 \%(2 / 39)$ \\
\hline Summation & & $5.5 \%$ & $5 \%$ & & $16.3 \%$ & $0 \%$ & $7.7 \%$ \\
\hline
\end{tabular}

*Mannucci \& Franchini. ${ }^{12}$

†GenoudV et al. ${ }^{13}$

‡lglesias Varela ML et al. ${ }^{14}$

§Rios M et al. 2016.

\section{Discussion}

Various works demonstrated the contribution of prothrombotic genetic polymorphisms on the risk of developing an event in both the venous and arterial beds. However it a higher prevalence of FVL was recorded in European Caucasians populations compared to Asian, African and Native Ameri Canos. ${ }^{10-12}$ The Argentine Cooperative Group for Hemostasis and Thrombosis studied the prevalence of these mutations in Argentina, with values similar to those reported for southern Europe. This study showed that of the 20 individuals studied in Tucumán, not a single one had a mutation for FVL and only one was heterozygous for PT20210 (Table 3). ${ }^{12,13}$ Although the population of Argentina is the result of the convergence of immigrants from various origins, mainly Spanish and Italian, the composition of the same is not homogeneous throughout the territory, presenting greater representativeness of the population in the northern and southern areas native. Previous studies carried out by our group on 39 women who had ET during a pregnancy did not record the presence of these mutations; while in the current study of women with ET outside of pregnancy we observed both genetic alterations but with a lower magnitude than that reported by another Argentine group from a medical center in the city of Buenos Air is Iglesias Varela ML. ${ }^{14}$

\section{Conclusion}

Local genetic variations should be considered in order to obtain useful information about the relative weight of hereditary and environmental risk factors for the thrombotic event in each region. This is an important tool in public health decision making. Knowing the prevalence of these polymorphisms (FVL and PT20210) in women from Tucumán who manifested a thrombosis provides knowledge when evaluating the pathophysiology of young patients, giving people the opportunity to modify habits to minimize environmental prothrombotic factors, thus decreasing the morbidity and mortality generated by this pathology.

\section{Acknowledgment}

None.

\section{Conflicts of interest}

There were no conflicts of interest during the study.

\section{Funding source}

Scholarship program "Dr. Abraam Sonis", In dividual category granted by the National Ministry of Health, through the National Health Research Commission.

\section{References}

1. Palmer S. Diagnostic and Treatment Guides. Argentina: Argentine Society of Hematology;2015. p. 750.

2. https://es.worldthrombosisday.org/

3. Consensus and Guides of the Argentine Federation of Cardiolog. 
4. Kyle P, Eichinger S. Deep Vein Thrombosis. Lancet. 2005;365(9465):1163-1174.

5. Prandoni P, Villalta S, Bagatella P, et al. The clinical course of deepvein thrombosis. prospective long-term follow-up of 528 symptomatic patients. Haematologica. 1997;82(4):423-428.

6. Bertina RM. Factor V Leiden and Prothrombin 20210 A. Common Risk aleles for Venous Thrombosis. ISH-EHA Combined haematology Congress 4-8 July. Amsterdam, Netherlands:Educational Program Book; 2019. p. 135-132.

7. Dahlback B. Resistance to activated Protein $\mathrm{C}$ caused by the factor V R506Q mutation is a common risk factor for venous thrombosis. Thromb Haemost 1997;242(S740):1-8.

8. Fundamentals for practical management in the Haemostasis Laboratory 2nd edn. Argentina: Argentine Cooperative Group of Hemostasis and Thrombosis; 2013.

9. Poort SR, Rosendaal FR, Reitsma PH, et al. A common genetic variation in the 3 'untranslated region of the prothrombin gene is associated with elevated plasma prothrombin levels and increased in venous thrombosis. Blood. 1996;88(10):3698-3703.
10. Koeleman BP, Reitsma PH, Allaart CF, et al. Activated protein C resistance as an additional risk factor for thrombosis in protection $\mathrm{C}$ deficient families. Blood. 1994;84(4):1031-1035.

11. Dzimiri N, Meyer B. World distribution of factor V Leiden. Lancet. 1995;347(8999):1133-1134.

12. Mannucci P, Franchini M. The real value of thrombophilia markers in identifying patients at high risk of VTE. Expert Rev Hematol. 2014;7(6):757-765.

13. Genoud V, Castañón M, Annichino-Bizzacchi J, et al. Prevalence of three Prothrombotic Polymorfisms:Factor V G1691A, Factor II G20210A and Metylentetrahydrofolate Reductase (MTHFR) C677T in Argentina. Thrombosi s Res. 2000;100(3):127-131.

14. Iglesias Varela ML, Adamczuk Y, Forastiero R, et al. Major and Potential Prothrombotic Genotypes in a Cohort of Patients With Venous Thromboembolism. Thromb Res. 2001;104(1):317-324. 